IJMMS 27:11 (2001) 675-680

PII. S0161171201010249

http://ijmms.hindawi.com

(c) Hindawi Publishing Corp.

\title{
AN EXPLICIT SOLUTION OF COUPLED VISCOUS BURGERS' EQUATION BY THE DECOMPOSITION METHOD
}

\author{
DOǦAN KAYA
}

(Received 12 March 2000 and in revised form 12 August 2000)

\begin{abstract}
We consider a coupled system of viscous Burgers' equations with appropriate initial values using the decomposition method. In this method, the solution is calculated in the form of a convergent power series with easily computable components. The method does not need linearization, weak nonlinearity assumptions or perturbation theory. The decomposition series solution of the problem is quickly obtained by observing the existence of the self-canceling "noise" terms where the sum of components vanishes in the limit.
\end{abstract}

2000 Mathematics Subject Classification. 35Q53.

1. Introduction. We consider the following coupled Burgers' equations:

$$
\begin{aligned}
& u_{t}-u_{x x}+u u_{x}+a(u v)_{x}=F(x, t), \\
& v_{t}-v_{x x}+v v_{x}+b(u v)_{x}=G(x, t),
\end{aligned}
$$

with initial conditions

$$
u(x, 0)=f(x), \quad v(x, 0)=g(x),
$$

for $0<x<1, t>0$. Here, $F(x, t), G(x, t)$ are given functions and $a, b$ are constants.

The coupled system is derived by Esipov [6]. It is a simple model of sedimentation or evolution of scaled volume concentrations of two kinds of particles in fluid suspensions or colloids, under the effect of gravity [7]. In this paper, the approximate solution of the coupled Burgers' equations, homogeneous or inhomogeneous, will be handled more easily, quickly, and elegantly by the Adomian's decomposition method $[1,2,3]$ than by the traditional methods for the exact solutions. To evaluate exact solutions for these problems, the decomposition scheme will be illustrated by studying suitable coupled system examples either homogeneous or inhomogeneous form. We will also illustrate the self-canceling phenomena for a inhomogeneous form of equations, using the decomposition method. Furthermore, we will show that considerably better approximations related to the accuracy level would be obtained if numerical solution is needed.

2. An analysis of the method. In this section, we outline the method to obtain approximate solutions of (1.1) and (1.2) using the decomposition method. We consider 
(1.1) in an operator form

$$
\begin{aligned}
& L_{t} u-L_{x x} u+N u+a K(u, v)=F(x, t), \\
& L_{t} v-L_{x x} v+M v+b K(u, v)=G(x, t),
\end{aligned}
$$

where the notations $N u=u u_{x}, M v=v v_{x}$, and $K(u, v)=(u v)_{x}$ symbolize the nonlinear terms, the notation $L_{t}=\partial / \partial t$ and $L_{x x}=\partial^{2} / \partial x^{2}$ symbolize the linear differential operators. Assuming that the inverse of the operator $L_{t}$ exists and that it can conveniently be taken as the definite integral with respect to $t$ from 0 to $t$, that is, $L_{t}^{-1}=\int_{0}^{t}(\cdot) d t$. Thus, applying the inverse operator $L_{t}^{-1}$ to (2.1) yields

$$
\begin{aligned}
& L_{t}^{-1} L_{t} u=L_{t}^{-1}\{F(x, t)\}-L_{t}^{-1} N u-a L_{t}^{-1} K(u, v)+L_{t}^{-1} L_{x x} u, \\
& L_{t}^{-1} L_{t} v=L_{t}^{-1}\{G(x, t)\}-L_{t}^{-1} M v-b L_{t}^{-1} K(u, v)+L_{t}^{-1} L_{x x} v,
\end{aligned}
$$

Therefore, it follows that

$$
\begin{aligned}
& u(x, t)=u(x, 0)+L_{t}^{-1}\{F(x, t)\}-L_{t}^{-1} N u-a L_{t}^{-1} K(u, v)+L_{t}^{-1} L_{x x} u, \\
& v(x, t)=v(x, 0)+L_{t}^{-1}\{G(x, t)\}-L_{t}^{-1} M v-b L_{t}^{-1} K(u, v)+L_{t}^{-1} L_{x x} v .
\end{aligned}
$$

We obtain the zeroth components by

$$
u_{0}=u(x, 0)+L_{t}^{-1}\{F(x, t)\}, \quad v_{0}=v(x, 0)+L_{t}^{-1}\{G(x, t)\},
$$

which is defined by all terms that arise from the initial conditions and from integrating the source terms. Then, decomposing the unknown functions $u(x, t)$ and $v(x, t)$ gives a sum of the components defined by the decomposition series

$$
u(x, t)=\sum_{n=0}^{\infty} u_{n}(x, t), \quad v(x, t)=\sum_{n=0}^{\infty} v_{n}(x, t) .
$$

Let the nonlinear terms $N u=u u_{x}, M v=v v_{x}$, and $K(u, v)=(u v)_{x}$ be expressed in the form of $A_{n}, B_{n}$, and $C_{n}$ Adomian's polynomials [3]; thus $N u=u u_{x}=\sum_{n=0}^{\infty} A_{n}$, $M v=v v_{x}=\sum_{n=0}^{\infty} B_{n}$, and $K(v, v)=(u v)_{x}=\sum_{n=0}^{\infty} C_{n}$ where $A_{n}, B_{n}$, and $C_{n}$ are the appropriate Adomian's polynomials which are generated forms of the following formula:

$$
\begin{aligned}
& A_{0}=\Psi\left(u_{0}\right) \\
& A_{1}=u_{1}\left(\frac{\partial}{\partial u_{0}}\right) \Psi\left(u_{0}\right) \\
& A_{2}=u_{2}\left(\frac{\partial}{\partial u_{0}}\right) \Psi\left(u_{0}\right)+\left(\frac{u_{1}^{2}}{2 !}\right)\left(\frac{\partial^{2}}{\partial u_{0}^{2}}\right) \Psi\left(u_{0}\right), \\
& A_{3}=u_{3}\left(\frac{\partial}{\partial u_{0}}\right) \Psi\left(u_{0}\right)+u_{1} u_{2}\left(\frac{\partial^{2}}{\partial u_{0}^{2}}\right) \Psi\left(u_{0}\right)+\left(\frac{u_{1}^{3}}{3 !}\right)\left(\frac{\partial^{3}}{\partial u_{0}^{3}}\right) \Psi\left(u_{0}\right),
\end{aligned}
$$

and so on. The Adomian's polynomials $B_{n}$ and $C_{n}$ are constructed as it was mentioned in $A_{n}$ polynomials. The remaining components $u_{n}(x, t)$ and $v_{n}(x, t), n \geq 1$, can be 
completely determined such that each term is computed by using the previous term. Since $u_{0}$ and $v_{0}$ are known,

$$
\begin{aligned}
u_{1} & =-L_{t}^{-1}\left(A_{0}\right)-a L_{t}^{-1}\left(C_{0}\right)+L_{t}^{-1} L_{x x}\left(u_{0}\right), \\
v_{1} & =-L_{t}^{-1}\left(B_{0}\right)-b L_{t}^{-1}\left(C_{0}\right)+L_{t}^{-1} L_{x x}\left(v_{0}\right), \\
u_{2} & =-L_{t}^{-1}\left(A_{1}\right)-a L_{t}^{-1}\left(C_{1}\right)+L_{t}^{-1} L_{x x}\left(u_{1}\right), \\
v_{2} & =-L_{t}^{-1}\left(B_{1}\right)-b L_{t}^{-1}\left(C_{1}\right)+L_{t}^{-1} L_{x x}\left(v_{1}\right), \\
& \vdots \\
u_{n} & =-L_{t}^{-1}\left(A_{n-1}\right)-a L_{t}^{-1}\left(C_{n-1}\right)+L_{t}^{-1} L_{x x}\left(u_{n-1}\right), \\
v_{n} & =-L_{t}^{-1}\left(B_{n-1}\right)-b L_{t}^{-1}\left(C_{n-1}\right)+L_{t}^{-1} L_{x x}\left(v_{n-1}\right),
\end{aligned}
$$

for $n \geq 0$. It is useful to note that the recursive relationship is constructed on the basis that the zeroth components $u_{0}(x, t)$ and $v_{0}(x, t)$ are defined by all terms that arise from the initial conditions and from integrating the source term. The remaining components $u_{n}(x, t)$ and $v_{n}(x, t), n \geq 0$, can be completely determined such that each term is computed by using the previous term. As a result, the components $u_{0}$, $u_{1}, u_{2}, \ldots$ and $v_{0}, v_{1}, v_{2}, \ldots$ are identified and the series solutions thus entirely determined. However, in many cases the exact solution in a closed form may be obtained. Furthermore, the method provides decomposition series solutions which generally converge very rapidly in physical problems. If the series converges with the $\gamma$-term partial sums, then

$$
\phi_{\gamma}=\sum_{k=0}^{\gamma-1} u_{k}(x, y), \quad \varphi_{\gamma}=\sum_{k=0}^{\gamma-1} v_{k}(x, y)
$$

can serve as a practical solution due to $\lim _{\gamma \rightarrow \infty} \phi_{\gamma}=u$ and $\lim _{\gamma \rightarrow \infty} \varphi_{\gamma}=v$ by definition $[2,5]$. We will see that $\gamma$ is generally very small.

As a result, the series solutions are given by

$$
\begin{aligned}
& u(x, t)=u_{0}-\sum_{n=1}^{\infty}\left\{L_{t}^{-1}\left(A_{n-1}\right)+a L_{t}^{-1}\left(C_{n-1}\right)-L_{t}^{-1} L_{x}\left(u_{n-1}\right)\right\} \\
& v(x, t)=u_{0}-\sum_{n=1}^{\infty}\left\{L_{t}^{-1}\left(B_{n-1}\right)+b L_{t}^{-1}\left(C_{n-1}\right)-L_{t}^{-1} L_{x}\left(v_{n-1}\right)\right\}
\end{aligned}
$$

where $L_{t}^{-1}$ is the previously given integration operator, $A_{n-1}, B_{n-1}$, and $C_{n-1}$ are defined appropriate Adomian's polynomials by (2.6). The solutions $u(x, t)$ and $v(x, t)$ must satisfy the requirements imposed by the initial conditions. The decomposition method provides a reliable technique that requires less work as compared with the traditional techniques.

Adomian and Rach [4] and Wazwaz [8] have investigated the phenomena of the self-canceling "noise" terms where the sum of components vanishes in the limit. An important observation they made was that "noise" terms appear for inhomogeneous cases only. The present author agrees with the previous authors' findings in case implementing the method for solving inhomogeneous equation. Further, it was formally 
justified in the last section that if terms in $u_{0}$ and $v_{0}$ are canceled by terms respectively in $u_{1}$ and $v_{1}$ even though $u_{1}$ and $v_{1}$ include further terms, then the remaining non-canceled terms in $u_{1}$ and $v_{1}$ are canceled by terms in $u_{2}$ and $v_{2}$, and so on. Finally, the exact solutions of the equations are readily found for the inhomogeneous case by determining the first two or three components of the solutions $u(x, t), v(x, t)$, and by keeping only the non-canceled terms of $u_{0}$ and $v_{0}$. To give a clear overview of the methodology, the following examples will be discussed.

\section{Implementations}

Problem 3.1. For the purposes of illustration of the decomposition method for solving the homogeneous form of a coupled Burgers' equations, we will consider the system of equations

$$
u_{t}-u_{x x}-2 u u_{x}+(u v)_{x}=0, \quad v_{t}-v_{x x}-2 v v_{x}+(u v)_{x}=0,
$$

the solutions of which are to be obtained subject to the initial conditions

$$
u(x, 0)=\sin (x), \quad v(x, 0)=\sin (x) .
$$

For the solution of this equation, we simply take the equation in an operator form, exactly in the same manner as the form of (2.2) and use (2.4) to find the zeroth components of $u_{0}$ and $v_{0}$ as

$$
u_{0}=\sin (x), \quad v_{0}=\sin (x)
$$

and obtain in succession $u_{1}, v_{1}, u_{2}, v_{2}$, and $u_{3}, v_{3}$, and so forth by using (2.7) with (2.6) to determine the other individual terms of the decomposition series, we find

$$
\begin{aligned}
u_{1} & =2 L_{t}^{-1}\left(u_{0} u_{0_{x}}\right)-L_{t}^{-1}\left(u_{0} v_{0}\right)_{x}+L_{t}^{-1} L_{x x}\left(u_{0}\right) \\
& =-t \sin (x), \\
v_{1} & =2 L_{t}^{-1}\left(v_{0} v_{0_{x}}\right)-L_{t}^{-1}\left(u_{0} v_{0}\right)_{x}+L_{t}^{-1} L_{x x}\left(v_{0}\right) \\
& =-t \sin (x), \\
u_{2} & =2 L_{t}^{-1}\left(u_{1} u_{0_{x}}+u_{0} u_{1_{x}}\right)-L_{t}^{-1}\left(u_{1} v_{0}+u_{0} v_{1}\right)_{x}+L_{t}^{-1} L_{x x}\left(u_{1}\right) \\
& =\frac{t^{2}}{2 !} \sin (x), \\
v_{2} & =2 L_{t}^{-1}\left(v_{1} v_{0_{x}}+v_{0} v_{1_{x}}\right)-L_{t}^{-1}\left(u_{1} v_{0}+u_{0} v_{1}\right)_{x}+L_{t}^{-1} L_{x x}\left(v_{1}\right) \\
& =\frac{t^{2}}{2 !} \sin (x), \\
u_{3} & =2 L_{t}^{-1}\left(u_{2} u_{0_{x}}+u_{1} u_{1_{x}}+u_{0} u_{2_{x}}\right)-L_{t}^{-1}\left(u_{2} v_{0}+u_{1} v_{1}+u_{0} v_{2}\right)_{x}+L_{t}^{-1} L_{x x}\left(u_{1}\right) \\
& =-\frac{t^{3}}{3 !} \sin (x), \\
v_{3} & =2 L_{t}^{-1}\left(v_{2} v_{0_{x}}+v_{1} v_{1_{x}}+v_{0} v_{2_{x}}\right)-L_{t}^{-1}\left(u_{2} v_{0}+u_{1} v_{1}+u_{0} v_{2}\right)_{x}+L_{t}^{-1} L_{x x}\left(v_{1}\right) \\
& =-\frac{t^{3}}{3 !} \sin (x),
\end{aligned}
$$


and so on, in this manner four components of the decomposition series were obtained, of which $u(x, t)$ and $v(x, t)$ were evaluated to have the following expansions:

$$
\begin{aligned}
& u(x, t)=u_{0}+u_{1}+u_{2}+u_{3}+\cdots=\sin (x)\left\{1-t+\frac{t^{2}}{2 !}-\frac{t^{3}}{3 !}+\cdots\right\}, \\
& v(x, t)=v_{0}+v_{1}+v_{2}+v_{3}+\cdots=\sin (x)\left\{1-t+\frac{t^{2}}{2 !}-\frac{t^{3}}{3 !}+\cdots\right\} .
\end{aligned}
$$

This expansion is exact to the last term as one can verify with some effort by expanding the appropriate solution of the coupled Burgers' equations (3.1), namely,

$$
u(x, t)=e^{-t} \sin (x), \quad v(x, t)=e^{-t} \sin (x) .
$$

This result can be verified through substitution.

Problem 3.2. As an example of the application of the self-canceling phenomena $[4,8]$, we seek the analytic solution of the inhomogeneous coupled Burgers' equations

$$
\begin{gathered}
u_{t}-u_{x x}+u u_{x}+(u v)_{x}=x^{2}-2 t+2 x^{3} t^{2}+t^{2} \\
v_{t}-v_{x x}+v v_{x}+(u v)_{x}=\frac{1}{x}-\frac{2 t}{x^{3}}-\frac{t^{2}}{x^{3}}+t^{2}
\end{gathered}
$$

subject to the initial conditions

$$
u(x, 0)=0, \quad v(x, 0)=0 .
$$

To obtain the decomposition solutions subject to the initial conditions given, we use (2.2) and (2.4) to determine the individual terms of the decomposition series, we get immediately

$$
\begin{aligned}
u_{0} & =x^{2} t-t^{2}+\frac{2 x^{3} t^{3}}{3}+\frac{t^{3}}{3}, \quad v_{0}=\frac{t}{x}-\frac{t^{2}}{x^{3}}-\frac{t^{3}}{3 x^{3}}+\frac{t^{3}}{3} \\
u_{1} & =-L_{t}^{-1}\left(u_{0} u_{0_{x}}\right)-L_{t}^{-1}\left(u_{0} v_{0}\right)_{x}+L_{t}^{-1} L_{x x}\left(u_{0}\right) \\
& =t^{2}-\frac{2 x^{3} t^{3}}{3}-\frac{t^{3}}{3}+\frac{3 x t^{4}}{2}-\frac{2 x^{4} t^{5}}{3}+\cdots, \\
v_{1} & =-L_{t}^{-1}\left(v_{0} v_{0 x}\right)-L_{t}^{-1}\left(u_{0} v_{0}\right)_{x}+L_{t}^{-1} L_{x x}\left(v_{0}\right) \\
& =\frac{t^{2}}{x^{3}}+\frac{t^{3}}{3 x^{3}}-\frac{t^{3}}{3}-\frac{4 t^{3}}{x^{5}}-\frac{t^{4}}{2 x^{2}}-\frac{2 t^{4}}{x^{5}}+\cdots,
\end{aligned}
$$

and similarly for higher terms. It is obvious that the self-canceling "noise" terms appear between various components, looking into the second, third, and fourth terms of $u_{0}$ and $v_{0}$ (3.9) and the first, second, and third terms of $u_{1}$ (3.10) and $v_{1}$ (3.11) are the self-canceling "noise" terms. We can readily observe that the fourth and the other terms in $u_{1}, v_{1}$ and the first, second, and the other terms in $u_{2}$ and $v_{2}$ are self-canceling "noise" terms, and so on. Keeping the remaining non-canceled terms and using (2.5) leads immediately to the solutions of (3.7) with initial conditions (3.8) given by

$$
u(x, t)=x^{2} t, \quad v(x, t)=\frac{t}{x}
$$


which can be verified through substitution. It is worth noting that noise terms between components of series will be canceled, and the sum of these "noise" terms will vanish in the limit. This has been justified by $[4,8]$.

4. Conclusions. In this paper, the Adomian decomposition method was used for homogeneous and inhomogeneous coupled Burgers' equations with initial conditions. It may be concluded that the Adomian methodology is a very powerful and efficient technique in finding exact solutions for wide classes of problems. With regard to this application, the decomposition method outlined in the previous sections finds quite practical analytic results with less computational work by using the Adomian's decomposition method. It is also worth noting to point out that the advantage of the decomposition methodology shows a fast convergence of the solution which may be achieved by observing the self-canceling "noise" terms.

Clearly, the series solution methodology can also be applied to other much more complicated nonlinear problems. However, we illustrated in the previous sections that the decomposition method does not require linearization or perturbation. Additionally, it does not make closure approximation, smallness assumptions or physically unrealistic white noise assumption in the nonlinear stochastic case [1, 2, 3].

\section{REFERENCES}

[1] G. Adomian, Nonlinear Stochastic Operator Equations, Academic Press, Florida, 1986. MR 88j:60112. Zbl 609.60072.

[2] _ A review of the decomposition method in applied mathematics, J. Math. Anal. Appl. 135 (1988), no. 2, 501-544. MR 89j:00046. Zbl 671.34053.

[3] _ Solving Frontier Problems of Physics: The Decomposition Method, Fundamental Theories of Physics, vol. 60, Kluwer Acad. Publ., Boston, 1994. MR 95e:00026. Zbl 802.65122.

[4] G. Adomian and R. Rach, Noise terms in decomposition solution series, Comput. Math. Appl. 24 (1992), no. 11, 61-64. CMP 1186 719. Zbl 777.35018.

[5] Y. Cherruault, Convergence of Adomian's method, Kybernetes 18 (1989), no. 2, 31-38. MR 90i:65109. Zbl 697.65051.

[6] S. E. Esipov, Coupled Burgers'equations: a model of polydispersive sedimentation, Phys. Rev. E 52 (1995), 3711-3718.

[7] J. Nee and J. Duan, Limit set of trajectories of the coupled viscous Burgers' equations, Appl. Math. Lett. 11 (1998), no. 1, 57-61. CMP 1490380.

[8] A. M. Wazwaz, Necessary conditions for the appearance of noise terms in decomposition solution series, J. Math. Anal. Appl. 5 (1997), 265-274.

DoĞan Kaya: Department of Mathematics, Firat University, Elazig 23119, Turkey

E-mail address: dkaya@firat.edu.tr 


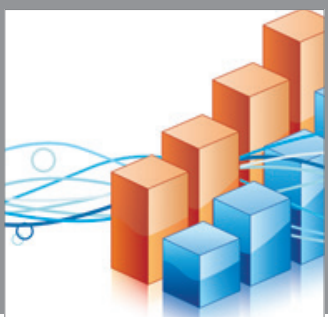

Advances in

Operations Research

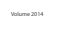

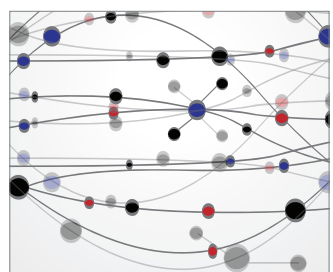

\section{The Scientific} World Journal
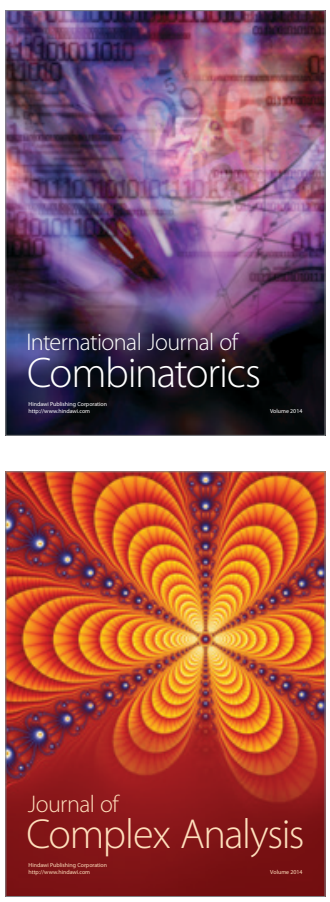

International Journal of

Mathematics and

Mathematical

Sciences
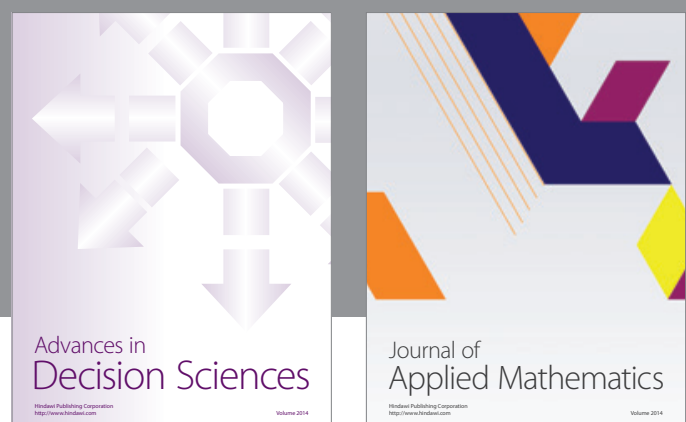

Journal of

Applied Mathematics
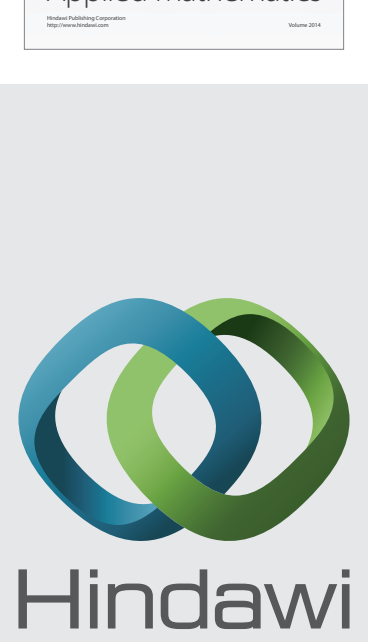

Submit your manuscripts at http://www.hindawi.com
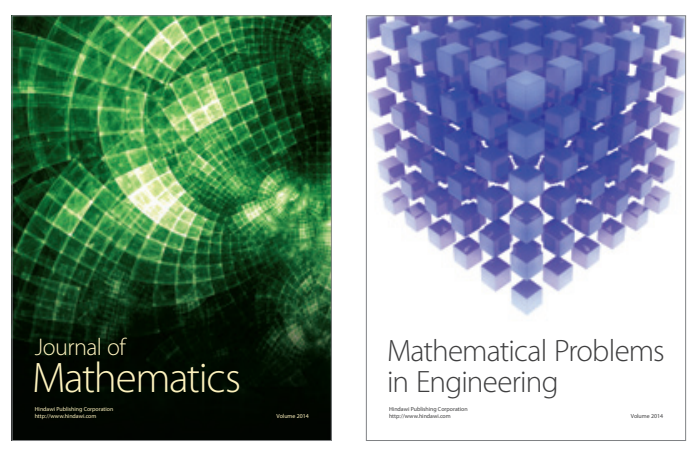

Mathematical Problems in Engineering
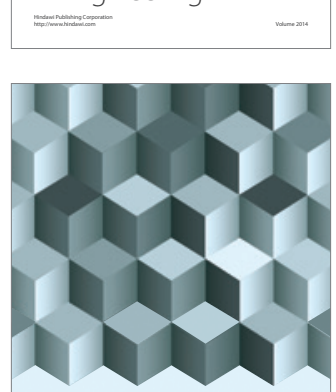

Journal of

Function Spaces
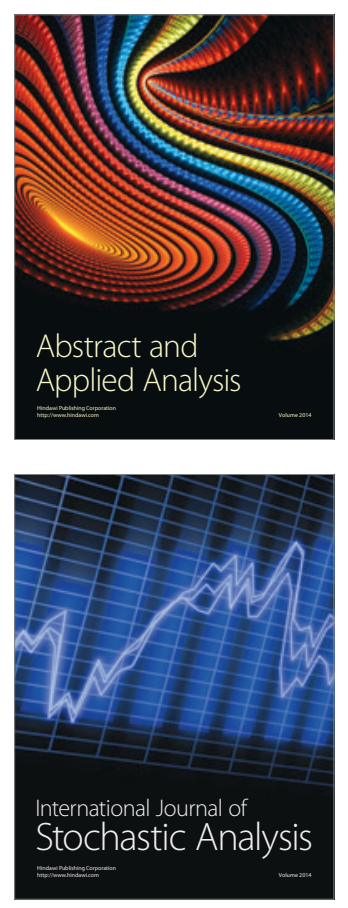

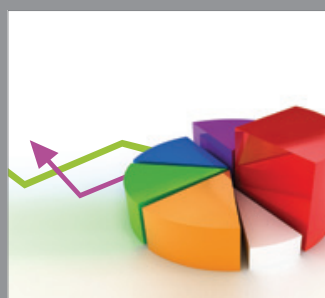

ournal of

Probability and Statistics

Promensencen
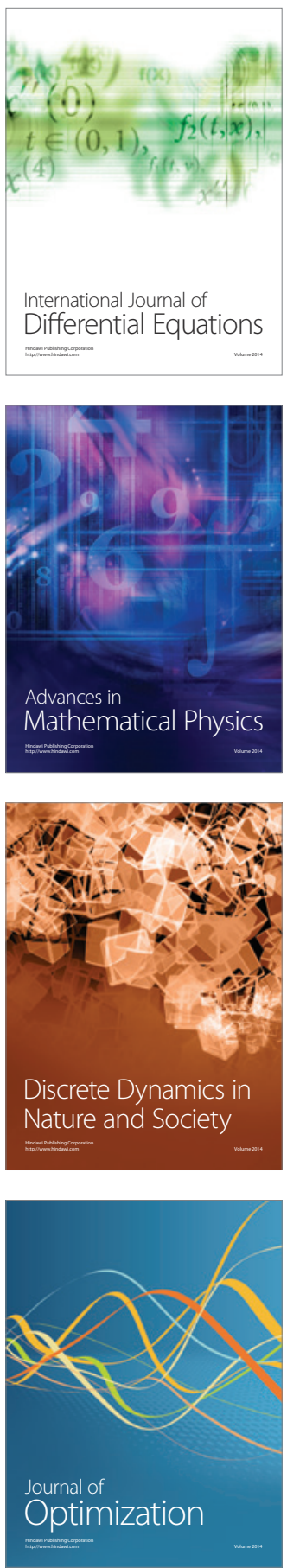